\title{
Collision of Two Highly Ionized Clouds of Gas
}

\author{
F. D. KAHN \\ Department of Astronomy, Manchester University, Manchester, England
}

1.

$I^{\mathrm{F}}$ $\mathrm{F}$ two rarefied masses of gas collide at a sufficiently high speed, their relative motion will be stopped by a collective plasma instability, rather than by collisions of individual particles. This is true even if the gases are initially non-ionized. In the present note, the possible influence of magnetic fields is ignored for the most part, but a criterion is found to decide under what conditions the collective interaction remains dominant even when they cannot be ignored. It seems that the neglect of magnetic effects may be justified, for example, in the case of two galaxies in collision at a relative speed of $1000 \mathrm{~km} / \mathrm{sec}$. The magnetic energy density here is $(1 / 8 \pi) H^{2} \sim 4 \times 10^{-12} \mathrm{erg} / \mathrm{cm}^{3}$ (with $H \sim 10^{-5}$ gauss), which is much less than the kinetic energy density $\frac{1}{2} N m U^{2} \sim 8 \times 10^{-9} \mathrm{erg} / \mathrm{cm}^{3}$ (with $N \sim 1$ particle per $\mathrm{cm}^{3}$ ).

\section{2.}

We deal first with the collision of two non-ionized gas clouds. For the sake of definiteness let us assume that both consist exclusively of $\mathrm{H}$ atoms, and that their relative speed is of the order of $1000 \mathrm{~km} / \mathrm{sec}$, as in the two colliding galaxies of the radio source Cygnus A (see, for instance, Baade and Minkowski. ${ }^{1}$ Two $\mathrm{H}$ atoms belonging to the two different clouds then have a relative energy of about $5 \mathrm{kev}$. The electrons, bound in the atoms with energies of $13.6 \mathrm{ev}$, can have little mechanical influence in a collision at such a high energy. Rather they will tend to be stripped off by some process such as

$$
H(1 s)+H(1 s)=H(1 s)+H^{+}+e,
$$

a reaction studied by Bates and Griffing. ${ }^{2}$ These authors calculated (see their Fig. 6) that the $\mathrm{H}$ atoms have a maximum cross section $0.6 \pi a_{0}^{2}$ for this reaction when the relative kinetic energy is about $25 \mathrm{kev}$. At $5 \mathrm{kev}$ relative energy the cross section is about $0.3 \pi a_{0}{ }^{2} .\left(\pi a_{0}{ }^{2}\right.$ $=8.8^{-17} \mathrm{~cm}^{2}$.)

If the clouds have densities of about one atom per $\mathrm{cm}^{3}$ the mean free path for these stripping collisions is about $3 \times 10^{16} \mathrm{~cm}=0.01 \mathrm{pc}$.

Bates and Griffing also found how much energy is given to the electrons and the protons. It can be seen from Fig. 8 of their paper that, in a collision at $5 \mathrm{kev}$ relative energy, only about a quarter of the electrons ejected acquire more than $6.8 \mathrm{ev}$, and only about onetwentieth acquire more than $13.6 \mathrm{ev}$. The protons

\footnotetext{
1 W. Baade and R. Minkowski, Astrophys. J. 119, 215 (1954).

2 D. R. Bates and G. Griffing, Proc. Phys. Soc. (London) A66, 961 (1953).
}

receive very little additional energy, of the order of $0.01 \mathrm{ev}$, or less.

Thus, after a relatively shallow interpenetration, the two counter-streaming clouds will have been completely ionized, but their relative motion will go on as before.

\section{3.}

The author has discussed elsewhere what might be expected to happen in a collision of two fully ionized clouds of gas. ${ }^{3}$ It was shown that the counter-streaming of the electrons is stopped first, within a distance of the order of $\left[m U^{2} / N \epsilon^{2}\right]^{2}$, where $N=$ number of protons (or electrons) per $\mathrm{cm}^{3}$ in each stream, $\epsilon=$ electronic charge, $m=$ electronic mass, and $2 U=$ relative velocity. With the assumed numerical values, $\left(m U^{2} / N \epsilon^{2}\right)^{\frac{1}{2}}=3$ $\times 10^{3} \mathrm{~cm}$. The relative motion of the electrons ceases almost at once, and is turned into plasma oscillations.

\section{4.}

We are now left with a stationary, oscillating electron gas, through which two streams of protons are moving in opposite directions. The solution of a set of nonlinear equations is needed to treat such a system properly, but this has not yet been found. It seems likely, however, that the motion will, once again, break up into irregular plasma oscillations. We shall deal only with the analogous linear problem of a small disturbance in an electron gas at rest with two groups of protons streaming through it.

Let there be $2 N$ electrons per $\mathrm{cm}^{3}$, and let there be $N$ protons per $\mathrm{cm}^{3}$ in each stream. Let the undisturbed velocities of the streams be $\pm U$ parallel to $O x$. Let $\epsilon$ be the charge of the proton, $-\epsilon$ the charge of the electron, $M$ the mass of the proton, and $m$ the mass of the electron. By the same method as in $\mathrm{Kahn}^{3}$ we find that, in the linear approximation, the electron gas obeys the equations

$$
\frac{\partial u_{0}}{\partial t}=-\frac{\epsilon}{m} E, \quad \frac{\partial s_{0}}{\partial t}+\frac{\partial u_{0}}{\partial x}=0,
$$

where $u_{0}=$ disturbance velocity of the electrons, $E=$ electrostatic field, and $s_{0}=$ fractional increase of electron density. The proton streams obey the relations

$$
\left(\frac{\partial}{\partial t} \pm U \frac{\partial}{\partial x}\right) u_{k}=\frac{\epsilon}{M} E, \quad\left(\frac{\partial}{\partial t} \pm U \frac{\partial}{\partial x}\right) s_{k}+\frac{\partial u_{k}}{\partial x}=0,
$$

where $k=1$ or 2 , the positive sign is taken for $k=1$ and

${ }^{3}$ F. D. Kahn, J. Fluid Mech. 2, 601 (1957). 


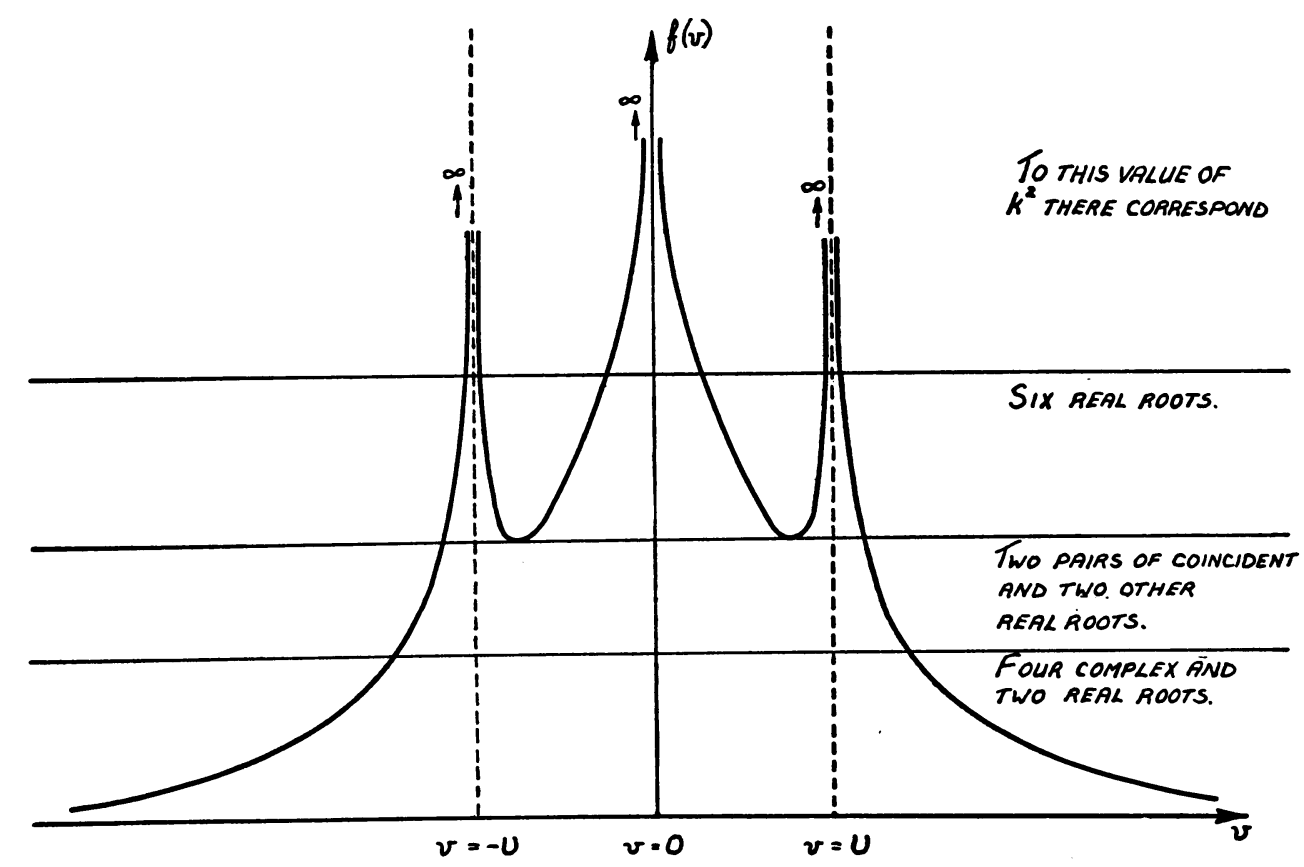

Fig. 1. To illustrate the occurrence of real and of complex roots in Eq. (2). the negative one for $k=2$, where $u_{k}=$ disturbance velocity in the $k$ th stream, and $s_{k}=$ fractional increase of proton density there. The electrostatic field is given by

$$
\partial E / \partial x=4 \pi N \epsilon\left(s_{1}+s_{2}-2 s_{0}\right) .
$$

On substitution of a trial solution

$$
s_{r}=a_{r} e^{i(\omega t-k x)}, \quad r=0,1,2,
$$

and on elimination of $a_{0}, a_{1}$, and $a_{2}$, we find that

$$
\frac{2 \Omega_{e}^{2}}{\omega^{2}}+\frac{\Omega_{p}^{2}}{(\omega-k U)^{2}}+\frac{\Omega_{p}^{2}}{(\omega+k U)^{2}}=1
$$

where $\Omega_{e}{ }^{2}=4 \pi N \epsilon^{2} / m$ and $4 \pi N \epsilon^{2} / M=\Omega_{p}{ }^{2}$. Equation (1) may also be written

$$
k^{2}=f(\omega / k) \equiv \frac{2 \Omega_{e}^{2}}{(\omega / k)^{2}}+\frac{\Omega_{p}^{2}}{[(\omega / k)-U]^{2}}+\frac{\Omega_{p}^{2}}{[(\omega / k)+U]^{2}}
$$

The system of charged particles is unstable if, for any real $k, \mathrm{Eq}$. (2) has a root $\omega / k$ with a negative imaginary part. The coefficients of Eq. (2) are real, and so its complex roots occur in pairs which are complex conjugates of one another. A plot of $f(v)$ against $v$ (Fig. 1) shows that, when $k^{2}$ is small enough, four of the six roots of Eq. (2) are complex, and so two roots have the property required for instability.

This result is easily extended. It may be shown that there is instability in any system in which the total charge per unit volume vanishes, and which consists of a finite number of uniform streams of charged particles, each moving with a given velocity.
5.

It is worth noting that a criterion may be found to decide whether the collective instability is at all important in stopping the relative motion, or whether a larger effect is due to collisions involving individual charged particles belonging to different streams. In Kahn, ${ }^{3}$ Sec. 2, it was shown that, when

$$
N \ll\left(m U^{2} / \epsilon^{2}\right)^{3},
$$

the collective instability predominates. [A numerical factor, insignificant for this argument, has been left out of (3).]

The inequality (3) can be given the following simple interpretation. The average distance between charged particles is of the order of $N^{-\frac{1}{3}}$; there is an electric field of the order of $E_{\mathrm{part}}=N^{\frac{3}{2} \epsilon}$ at this distance from a charged particle.

On the other hand the collective instability can build up a field $E_{p l}$ whose magnitude is at most such that

$$
E_{p l}{ }^{2} / 8 \pi \sim N m U^{2} / 2
$$

The inequality (3) may equally well be written

$$
N^{2} \epsilon \ll\left(N m U^{2}\right)^{\frac{1}{2}}
$$

or

$$
E_{\text {part }} \ll E_{p l} \text {. }
$$

This inequality shows that the collective instability is important if there is enough kinetic energy in the counter-streaming to build up a plasma field $E_{p l}$ whose strength, at an average point, is much greater than the field there due to the nearest charged particle.

A very rough argument can be used to extend this 
criterion to include the case in which there is present a magnetic field capable of being amplified. The greatest field intensity which the plasma can generate is of the order of

$$
H_{p l} \sim\left(N m U^{2}\right)^{\frac{1}{2}} .
$$

A charge $\epsilon$ moving through such a field at a speed $U$, experiences a deflecting force of the order of $\epsilon U H_{p l} / c$ and therefore acts as though it were exposed to an electric field $U H_{p l} / c$. The collective instability will be important if

or

$$
\begin{gathered}
E_{\text {part }} \ll U H_{p l} / c, \\
N \ll\left(\frac{m U^{2}}{\epsilon^{2}}\right)^{3}\left(\frac{U}{c}\right)^{6} .
\end{gathered}
$$

The upper limit for $N$ is now rather smaller, but it may still be large enough in many astronomical phenomena where high speeds $U$ and low densities $N$ occur.

\section{DISCUSSION}

E. C. BullaRD, Cambridge University, Cambridge, England: Is this a one-dimensional calculation?

F. D. KAHN, Manchester University, Manchester, England: Yes.

L. SPITZER, JR., Princeton University Observatory, Princeton, New Jersey: What was taken into account in the dispersion relations for the electron plasma oscillations. Were the positive ions ignored and two streams of interpenetrating electrons assumed?

\section{F. D. KAHN : Yes.}

L. SPITZER, JR. : And do you take into account the velocity dispersion of the electrons?

F. D. KAHN : No. The assumption is definitely that the dispersion of the electron velocities is small in comparison to the relative velocities.

L. SPITZER, JR. : How legitimate is that? Are not the two of the same order of magnitude? I would suppose that the velocity spread might have quite a large effect on the dispersion relation.

F. D. KAHN : That all depends on the relative speed of the collisions, and on the ambient temperature of the gas. One can imagine cases where it is legitimate. This example is just on the border line.

A. SCHLÜTER, Max Planck Institut für Physik, Göttingen, Germany: I should like to repeat a comment on the role of the magnetic field, that I made on several occasions before. The question is whether it is really reliable to estimate the importance of the magnetic field by just considering its energy density as compared to the energy densities of the fluid motions. If we have a mixture of the charged gases as we have here, or as we have in the case in which we want to confine a gas of cosmic rays (a gas of relativistic particles) in a given region, the conductivity of the single charge constituent of the gas is so high that, even if the magnetic field is very low, we cannot have relative motion of the gas perpendicular to the lines of force, but only motion along the lines of force, irrespective of the energy density of the gas. Thus, in a case like this, a magnetic field of very low strength would prevent one proton gas having a different velocity from the protons flowing in the other direction, provided that the magnetic field is perpendicular to the velocities, because the component of velocity across the lines of force must be the same for both constituents. This always holds if the gyration radius in the magnetic field is small compared to the distances considered. It applies as well to the argument which was put forward yesterday by Pickelner, and it also applies, for instance, to the argument used by Oort in evaluating the strength of the magnetic field within the Crab Nebula. In all these cases you cannot say a magnetic field will act on the charged particles efficiently only if the energy density of the magnetic field is sufficiently high. The magnetic field acts simply as a coupling agent between the different charged gases, and what you have to consider is really the total balance of momentum between all charged constituents together with the magnetic field.

F. D. KAHN : Would it not be possible for the kinetic energy density to be so high that the magnetic field is pushed out of some regions of the gas? I am thinking in analogy of a suggestion made by Chapman and Ferraro about the way corpuscular streams leave the sun. If they have to leave through the solar atmosphere and pass through the magnetic field there, a hole is blown in the magnetic field which is compensated by currents running on the boundaries. Would that not be possible in general?

A. SCHLÜTER : If you start originally with a situation where the lines of force penetrate through the gas, you cannot have that case, because the conductivity is so high that the lines of force are taken along. On the contrary, if you start with a gas which does not contain any magnetic fields - say you produce a plasma by expansion of an originally cool gas - then indeed you get that effect. 
E. Schatzman, Institut d'Astrophysique, Paris, France: You have neglected the magnetic field, but do you not think that in a collision there will be an amplification of the magnetic field?

M. P. SAVEDOFF, University of Rochester, Rochester, New York: I will report this afternoon on this point. I have used the de Hoffman-Teller shock relations including the magnetic field for this particular case and find that the amplification of the magnetic field (the field itself, not the pressure) is by a factor of four. I made the assumption finally that the magnetic field was a hundred times the gas pressure and went through the three equations of continuity of mass, momentum, and energy and ended up with essentially the results of Kahn.

F. D. KAHN : It is no more difficult to write down the equations with a vector potential and to see whether the rotational parts of the magnetic field can be amplified. You find that the amplification in this counterstreaming only occurs for the irrotational part; the rotational part of the vector potential is not amplified. There may, however, be more complicated effects which are ignored there.

H. K. SEN, GRD, AFCRC, Hanscom Field, Bedford, Massachusetts: I guess Savedoff is discussing a linear theory. If so, what is the frequency band width of amplification? In view of the high magnitude of the amplification I believe a nonlinear investigation would be required. In a nonlinear theory two types of effects would arise. One is that the nonlinear terms would cut down the amplitude and give you a maximum amplitude of amplification. The time constant derived from this treatment might be considerably different from what is obtained in a linear theory. The second type of effect is a frequency amplitude relation: the maximum amplitude would depend upon the frequency. The oscillation would be, I guess, very much anharmonic. In other words, you would not only observe a fundamental frequency, but you might observe higher harmonics which might be comparable to the funda- mental. Would Kahn expect any types of these effects in the present situation?

F. D. KAHN : Nonlinear effects certainly occur and the amplification must cease when the energy of oscillation of the electrons is comparable with their initial relative kinetic energy. What I am afraid of, in the case of the proton collision, is that the electrons which have to be treated nonlinearly, move around so fast that they can jump into the regions where there is an excess charge of protons and neutralize it very quickly. This would slow down the amplification very much more than anything else.

R. LANDSHOFF, Lockheed Aircraft Corporation, Sunnyvale, California: Is the idea that electrons, as they come in, pile up, that the cloud of electrons in the middle grows larger and larger?

\section{F. D. KAHN : Yes.}

H. C. VAN DE HULST, Leiden Observatory, Leiden, Netherlands: The Cygnus A source is one of the strongest radio sources we see from here, and yet it is a hundred million light years away. The theory as Kahn has presented it so far seems to mean that the electrons collide and stay where they are, and then the protons also collide and stay where they are, all within a distance of a few kilometers. What will happen then? Do you get shock waves?

A. R. KaNTROWITZ, Avco Research Laboratories, Everett, Massachusetts: I would like to talk at this point about the unimportance of the magnetic field, assuming that we start with the field energy small compared to the kinetic energy. If one supposes that before the clouds get in contact the field lines are attached to but extend outside the gas, then these field lines would simply be compressed between the two gases and the field energy will go up indefinitely so long as there are field lines coming away from each of the gas masses. This is not yet a shock wave, so it is not true that the field strength is thereby increased by some factor; it is an isotropic compression of the field between two conducting objects. 